\title{
Biomarkers of IL-33 and sST2 and Lack of Association with Carvedilol Therapy in Heart Failure
}

This article was published in the following Dove Press journal: Clinical Pharmacology: Advances and Applications

\author{
Negar Firouzabadi (iD) ${ }^{1-3}$ \\ Maryam Dashti (D) \\ Ali Dehshahri ${ }^{4}$ \\ Ehsan Bahramali ${ }^{5}$ \\ 'Department of Pharmacology and \\ Toxicology, School of Pharmacy, Shiraz \\ University of Medical Sciences, Shiraz, Iran; \\ ${ }^{2}$ Pharmaceutical Sciences Research Center, \\ Shiraz University of Medical Sciences, \\ Shiraz, Iran; ${ }^{3}$ Noncommunicable Diseases \\ Research Center, Fasa University of Medical \\ Sciences, Fasa, Iran; ${ }^{4}$ Department of \\ Pharmaceutical Biotechnology, Shiraz \\ University of Medical Sciences, Shiraz, Iran; \\ ${ }^{5}$ Digestive Disease Research Center, \\ Digestive Disease Research Institute, \\ Shariati Hospital, Tehran University of \\ Medical Sciences, Tehran, Iran
}

Objective: The IL-33/ST2 pathway plays a fundamental role in the cardiovascular system and can be considered as a new therapeutic strategy for the treatment or prevention of cardiovascular diseases. ST2, as an interleukin (IL)-1 receptor family member, has transmembrane (ST2L) and soluble (sST2) isoforms. sST2 neutralizes IL-33 and thereby inhibits the cardioprotective role of IL-33/ST2L signaling pathway. Increase in sST2 level is associated with weak cardiac output and can be a predictor of mortality in heart failure (HF). Thereby, we hypothesized that there may be a relationship between the cardioprotective effects of carvedilol and sST2 and IL-3 in HF patients.

Methods: sST2 and IL-33 were measured in serum of 66 individuals; 22 healthy volunteers and 44 suffering from HF; among whom 25 patients received carvedilol and the other 19 patients did not receive any $\beta$-blockers.

Results: Lack of association between serum levels of IL-33 and sST2 was observed between HF patients and healthy individuals $(2.4466 \pm 0.69$ vs $2.6748 \pm 0.33$ and $3416.6 \pm 1089.1$ vs $2971.6 \pm 792.5$, respectively). Our results indicated no significant difference between sST2 and IL-33 levels in HF patients who did not receive beta-blockers and patients receiving carvedilol ( $\mathrm{P}=0.59$ and $\mathrm{P}=0.97)$.

Conclusion: Our results showed a lack of association between serum levels of IL-33 and sST2 and HF. Moreover, the results do not confirm the cardioprotective mechanism of carvedilol by means of IL-33/sST2 pathway.

Keywords: heart failure, IL-33, sST2, carvedilol, $\beta$-blocker, biomarker

\section{Introduction}

The American Heart Association (AHA) describes heart failure (HF) as a complex clinical condition due to the inability of the heart to pump adequate blood to meet the metabolic needs of the body. This illness can be caused by structural disruption or dysfunction in the ventricular filling or ventricular contraction. In developed countries, coronary artery disease (CAD) is the main cause of HF in both genders and is responsible for $60-70 \%$ of HF cases. Seventy-five percent of patients with hypertension are also diagnosed with HF which often includes CAD patients. ${ }^{1}$ High blood pressure, CAD, diabetes mellitus ${ }^{1,2}$ and Myocardial Infarction (MI) are among the factors which increase the chance of HF. ${ }^{1}$

Neurohormonal hypothesis is now one of the best justifications for the pathophysiology of HF. The activation of endogenous neurohormones, including norepinephrine, angiotensin II, aldosterone, vasopressin, and a number of pre-inflammatory
Correspondence: Negar Firouzabadi Department of Pharmacology and Toxicology, School of Pharmacy, Shiraz University of Medical Sciences, Shiraz,

Tel +98-9|7-314-5303

Fax +98-7I3-2424I28

Email nfirouzabadi@yahoo.com 
cytokines play an important role in cardiac remodeling and thus progression of HF. Drugs used to modulate neurohormonal activity may hinder the progression of $\mathrm{HF}$ and reduce mortality rate $^{3}$.

Drugs such as angiotensin-converting enzyme (ACE) inhibitors, Angiotensin II Receptor Blockers (ARBs) and $\beta$-blockers are widely used in different stages of HF. Evidences support the positive effect of these drugs in survival of HF patients.

$\beta$-Blockers decrease cardiac work and reduce morbidity as well as mortality rate in mild-to-moderate cases of HF. Therapy with carvedilol, the $\beta$-Blocker of choice in $\mathrm{HF}$, not only increases life expectancy of these patients, but also improves clinical outcomes by reducing hospitalization and preventing arrhythmic complications in symptomatic $\mathrm{HF}^{4}$

Interleukin 33 (IL-33) is an intracellular nuclear factor that focuses on the nucleus by its terminal amines and can be attached to the heterochromatins. ${ }^{5}$ Human IL-33 gene located on chromosome 9, codes for 270 amino acids. ${ }^{6}$ Although the physiological role of IL-33 as a nuclear factor is not fully understood, it seems that this cytokine is involved in transcriptional regulation by binding to nucleosomes and regulating the density of chromatins. ${ }^{7}$ Fibroblasts, epithelial and endothelial cells, especially endothelial venules are the main sources of expressing IL-33. ${ }^{6}$ Lower expression levels of IL-33 were found in lymph tissue, spleen, pancreas, kidney, and heart. ${ }^{8}$ IL-33 as a new member of the IL-1 family exerts its effects by binding to its receptor ST2. ST2 is a member of the IL-1R/TLR superfamily with three isoforms: soluble form, sST2, which is expressed in embryonic tissues, mammary tumors, and fibroblasts. ${ }^{9}$ Trans membrane form ST2L, a membrane-anchored long form, which is restricted to the surface of T-helper 2 (Th2) cells and mast cells ${ }^{10}$ and Variant form, ST2V, which is expressed mainly in gastrointestinal organs such as stomach, large and small intestine, and spleen. $^{11}$

IL-33 increases Th2-dependent immune responses by binding to its dimeric receptor, and thus exerts its biological activity. This receptor is a complex of ST2L and receptor accessory protein. ${ }^{6}$ sST2 prevents IL-33 and ST2L interactions and limits the biological activity of IL33. IL-33 has a dual role in different diseases. By promoting Th2 immune response, it reduces atherosclerosis, ${ }^{6}$ obesity $^{12}$ and cardiac remodeling. ${ }^{8}$ Contrariwise it develops diseases such as asthma, atopic dermatitis, and anaphylaxis. ${ }^{6}$ Accordingly, the IL-33/ST2 pathway plays a fundamental role in the cardiovascular system ${ }^{13-16}$ and can be considered as a new therapeutic strategy for the treatment or prevention of cardiovascular diseases. ${ }^{8}$ It has been pointed out repeatedly that the signal derived from the IL-33/sST2 complex may affect fibroblasts and myocytes function in the heart and exert cardioprotective properties. $^{17}$

According to all the explanations given, this hypothesis raised that one of the mechanisms of carvedilol in HF may be due to its effect on the IL-33/sST2 pathway. Our study further investigated the aforementioned hypothesis.

\section{Materials and Methods}

\section{Sample Collection}

This study was carried out in accordance with The Code of Ethics of the World Medical Association (Declaration of Helsinki) and Uniform Requirements for manuscripts submitted to biomedical journals and was approved by the local committee for ethics of medical experiments on human subjects of Shiraz University of Medical Sciences with the ethical approval number of 15,870 . We obtained written consent from all participants prior to the interview. We used the infrastructure of a local hospital-based disease registry system FaRSH which has enrolled patients admitted with a diagnosis of systolic HF since July 2015. The main inclusion criterion was left ventricular ejection fraction (LVEF) of $<50 \%$. Fifty-five patients with diagnosis of systolic HF secondary to ischemic heart disease were recruited in the study. Patients were matched according to age, sex and body mass index (BMI). Those with severe valvular heart diseases, GFR $<30 \mathrm{~mL} / \mathrm{min}$ and significant comorbidities were excluded in the first place.

A total of 66 individuals comprised 22 healthy subjects and 44 patients diagnosed with HF were enrolled in our study. Among $44 \mathrm{HF}$ patients, 25 individuals (case group) received carvedilol for at least three months, the dose of which ranged from $6.25 \mathrm{mg}$ to $25 \mathrm{mg}$ in twice daily. $\beta$ blockers were contra indicated in the other 19 patients (control group) due to severe sinus bradycardia and partial atrioventricular (AV) block.

The exclusion criteria for healthy individuals was the absence of cardiovascular diseases, HTN, hyperlipidemia, diabetes, malignancies, and autoimmune, neurologic and psychiatric diseases. All healthy participants were nonsmokers.

Blood samples were taken and remained at room temperature for 2 hours. Thereafter, they were centrifuged for 
10 minutes at $3500 \mathrm{rpm}$ at $2-8^{\circ} \mathrm{C}$. The supernatants were stored at $-20^{\circ} \mathrm{C}$ for further analysis.

\section{Measurement of Serum IL-33 and sST2} Human sST2 (soluble ST2) ELISA Kit ${ }^{18}$ from Elabscience Company and ab119547- IL-33 Human ELISA Kit ${ }^{19}$ from Abcam Company were used to measure the level of IL-33 and sST2.

Human sST2 (soluble ST2) ELISA Kit is based on Sandwich-ELISA principle. Samples and standards were added to the micro ELISA plate wells pre-coated with Human sST2 antibody and combined with the specific antibody. Thereafter, Avidin-Horseradish Peroxidase (HRP) conjugate and a biotinylated detection antibody specific for Human sST2 were added sequentially to each well and incubated. Free components were washed away. The next step was adding the substrate solution to each well. Wells containing Human sST2, biotinylated detection antibody and Avidin-HRP conjugate appear blue in color. In order to terminate the enzyme-substrate reaction the stop solution was added to each well and the color turns yellow. The optical density (OD) was measured using UV spectrophotometer at a wavelength of $450 \mathrm{~nm}$.

For IL-33 assay, samples and standards were added to each well pre-coated with IL-33 specific antibodies and incubated at room temperature. Following washing, a Biotin-conjugated anti-Human IL-33 detection antibody was added to each plate and incubated again at room temperature. After washing, Streptavidin-HRP conjugate was added to each well, incubated at room temperature and washed again. The last step was adding TMB to each well to be catalyzed by HRP in order to produce a blue color product. By adding acidic stop solution, the color changes into yellow which will be detected at a wave length of $450 \mathrm{~nm}$ using UV-spectrophotometer.

\section{Statistical Analysis}

Continuous variables were reported as Mean \pm SD. Normality of the data was analyzed by KolmogorovSmirnov test. Continuous variables were compared using a parametric test (Student's $t$-test). Since both $\mathrm{sST} 2$ and IL-33 had normal distribution two-tailed Student's $t$-test was used to compare the mentioned parameters between different groups of our study. For the comparison of proportions, the $\chi 2$ test was used, with Yates or Fisher's corrections applied when needed. In order to omit the role of confounders, linear regression analysis was performed. For calculating the concentrations, logarithmic
Table I Demographic Characteristics of Healthy Individuals

\begin{tabular}{|l|l|}
\hline Characteristics & Healthy Individuals $(\mathbf{n}=22)$ \\
\hline Age (years) & $65.2 \pm 10.2$ \\
Male/female $(\mathrm{n} / \mathrm{n})$ & $1 \mathrm{I} / \mathrm{II}$ \\
$\mathrm{BMI}\left(\mathrm{kg} / \mathrm{m}^{2}\right)$ & $23.2 \pm 0.9$ \\
\hline
\end{tabular}

graphs in Excel 2013 were used. All statistics were conducted using SPSS, version 22.0. $\mathrm{P}$ values $<0.05$ were considered statistically significant.

\section{Results}

A total of 66 Individuals comprised 22 healthy subjects (male/female: 11/11; age: 65.2 \pm 10.2 ; BMI: $23.2 \pm 0.9$ ) and 44 patients diagnosed with HF (male/female: 24/20; age: 66.0 \pm 11.1 ; BMI:24.4 \pm 0.6 ) were enrolled in our study. Demographical characteristics of healthy subjects are demonstrated in Table 1. Demographical and clinical characteristics of HF subjects are presented in Table 2. No significant difference was observed between two groups regarding different parameters $(\mathrm{P}>0.05)$. Tables 3 and 4 show serum concentrations of IL-33 and sST2. Lack of association between serum levels of IL-33 and sST2 was observed comparing HF patients with healthy individuals. $(2.45 \pm 0.69$ vs $2.67 \pm 0.33$ and $3416.6 \pm 1089.1$ vs 2971.6 \pm 792.5 , respectively). Comparing HF patients treated with carvedilol and non-treated patients, serum sST2 levels showed no significant difference $(\mathrm{P}=0.59)$. Regarding IL33 , our results showed no significant difference between the two mentioned groups $(\mathrm{P}=0.97)$.

Table 2 Characteristics of Patients Receiving Carvedilol (Case) and Patients Not Receiving Carvedilol (Control)

\begin{tabular}{|c|c|c|c|}
\hline Characteristics & Case $(n=25)$ & Control $(n=19)$ & P-value \\
\hline Age (years) & $64.83 \pm 11.03$ & $67.25 \pm 11.06$ & 0.44 \\
\hline Male/female $(\mathrm{n} / \mathrm{n})$ & $14 / 11$ & $10 / 9$ & 0.533 \\
\hline BMI $\left(\mathrm{kg} / \mathrm{m}^{2}\right)$ & $24.3 \pm 0.5$ & $24.5 \pm 0.8$ & 0.756 \\
\hline Diabetes mellitus $(\mathrm{N}, \%)$ & $10(40.0 \%)$ & 8 (42.1\%) & 0.566 \\
\hline Smoking (N, \%) & $8(32.0 \%)$ & $7(36.8 \%)$ & 0.492 \\
\hline HTN (N,\%) & II (44.0\%) & $12(63.1 \%)$ & 0.434 \\
\hline LVEF (mean $\pm S D)$ & $33.6 \pm 1.68$ & $30.8 \pm 2.72$ & 0.35 \\
\hline AF $(N, \%)$ & $4(16.0 \%)$ & I (5.3\%) & 0.270 \\
\hline Heart valve disease $(\mathrm{N}, \%)$ & $4(16.0 \%)$ & $3(15.8 \%)$ & 0.657 \\
\hline $\mathrm{DCM}(\mathrm{N}, \%)$ & $3(12.0 \%)$ & $3(15.8 \%)$ & 0.525 \\
\hline Hyperlipidemia (N,\%) & $14(56.0 \%)$ & $10(52.6 \%)$ & 0.532 \\
\hline Stroke $(\mathrm{N}, \%)$ & I (4.0\%) & I (5.3\%) & 0.683 \\
\hline Previous MI (N, \%) & $13(56.0 \%)$ & II (58.0\%) & 0.467 \\
\hline History of malignancy $(\mathrm{N}, \%)$ & $2(8.0 \%)$ & $2(10.5 \%)$ & 0.585 \\
\hline
\end{tabular}

Abbreviations: SD, standard deviation; HTN, hypertension; LVEF, left ventricular ejection fraction; AF, atrial fibrillation; DCM, dilated cardiomyopathy. 
Table 3 sST2 and IL-33 Levels in Healthy and Heart Failure (HF) Subjects

\begin{tabular}{|c|c|c|c|}
\hline Parameter & Healthy Group (Mean \pm SD) & HF Group (Mean \pm SD) & P-value \\
\hline sST2 concentration & $2971.6 \pm 792.5$ & $3416.6 \pm 1089.1$ & 0.064 \\
\hline IL-33 concentration & $2.6748 \pm 0.33$ & $2.4466 \pm 0.69$ & $0.08 I$ \\
\hline
\end{tabular}

Table 4 sST2 and IL-33 Levels in HF Patients Treated with Carvedilol (Case) and Patients Not Receiving Any $\beta$ Blockers (Control)

\begin{tabular}{|c|c|c|c|}
\hline Parameter & Case (Mean \pm SD) & Control (Mean \pm SD) & P-value \\
\hline sST2 concentration & $3493.8 \pm 1083.3$ & $3315.1 \pm 1117.9$ & $P=0.59$ \\
\hline IL-33 concentration & $2.42 \pm 0.86$ & $2.47 \pm 0.30$ & $P=0.97$ \\
\hline
\end{tabular}

\section{Discussion}

Since the discovery of sST2 and its natural ligand, IL-33 in early 2000, attention was paid to the signaling cascade of IL-33/ST2. Some studies in animal models concerning this cascade have been performed but details regarding IL-33 and sST2 levels in HF patients treated with $\beta$ blockers such as carvedilol have not been reported to date. In many studies, the benefits of $\beta$-blocker therapy in HF have been well documented. ${ }^{4,20,21} \beta$-blockers have effects on beta-adrenergic receptors and inhibit the sympathetic system, thereby preventing the progression of HF by controlling cardiac remodeling. ${ }^{22}$ As it is known, sST2 is produced by cardiac fibroblasts and cardiomyocytes under conditions of cardiac damage and plays a significant role in cardiac remodeling. ${ }^{23}$ SST2 function is achieved by binding to its ligand, IL-33 which is produced by cardiac fibroblasts. IL-33 inhibits hypertrophy of cardiomyocytes, fibrosis and apoptosis. ${ }^{17,24}$ Probability of the effect of $\beta$-blocker therapy on IL-33/sST2 complex is still not fully understood.

Based on previous reports, we hypothesized that there may be a relationship between the cardioprotective effects of carvedilol and the two parameters of SST2 and IL-33.

Regarding the function of sST2, an increase in sST2 level is reported to be associated with weak cardiac output and left ventricular function. Thus, its level can be a predictor of mortality risk in cardiovascular patients or HF patients. ${ }^{6,16}$

Comparison of serum sST2 level between HF patients and healthy individuals in our study showed lack of association with HF $(\mathrm{P}=0.064)$. Previous studies that solely measured plasma SST2 in patients with cardiovascular diseases reported a significant increase in this parameter. It is notifiable that patients enrolled in previous studies had sudden cardiovascular incidence and did not have any mild and stable conditions. For example, serum sST2 level increases in acute MI (AMI) patients 1 day after stroke ${ }^{25}$ and sST2 levels predict probable deaths and HF in patients who had been admitted with MI. ${ }^{26,27}$ Also, the level of sST2 predicts the recovery effects of left ventricular function and remodeling after MI. ${ }^{28}$ In fact, many studies measured the level of this parameter in various cardiovascular diseases, most of which emphasized on the changes in this parameter after a cardiovascular event. In this regard the percentage of changes in the level of SST2 in HF patients might predict the occurrence of sudden events and even mortality. A report suggests that SST2 levels are lower in patients with mild HF who have not had sudden cardiac events. ${ }^{29}$ Patients with systolic HF represent higher serum levels of sST2 compared with patients confronting mild cardiac failure with preserved ejection fraction. ${ }^{30}$ Our results might be justifiable by the fact that enrolled patients in our study although suffered from HF, but did not have any sudden cardiac events and represented a milder condition, therefore did not exhibit high enough levels of sST2. Therefore, the difference regarding sST2 levels between HF patients and healthy subjects was not statistically significant. However, serial measurements of serum levels of sST2 would have further helped predicting and assessing the status of these patients.

Our study also showed lack of association between serum levels of IL-33 and HF. Studies have shown that IL-33 has a dual role in various diseases such as evoking Th2 immune response, reducing atherosclerosis ${ }^{6,31}$ and having a protective effect on cardiac remodeling ${ }^{8}$ and cardiac diseases. ${ }^{17}$ Contrariwise, an increase in plasma levels of IL-33 may be indicative of an autoimmune or a chronic disease. ${ }^{32}$ So signals related to IL-33 pathway can have both pro-inflammatory and protective properties. ${ }^{31}$ IL-33 also has the ability to inhibit angiotensin II and phenylephrine, which can cause cardiac hypertrophy and increase generation of reactive oxygen spices (ROS) in cardiomyocytes. ${ }^{17}$ It may be postulated that 
complications and morbidity observed in HF patients may be due to decreased levels of IL- $33^{33}$ which was not confirmed by our results. It is worth mentioning that the negative results achieved might be due to the small sample size of enrolled patients.

Among the study individuals, 16 patients did not receive any $\beta$-blockers, and 25 patients were carvedilol recipients. There was no statistically significant difference between the serum levels of IL-33 between these groups. But it is worth noting that the highest serum level of IL-33 in recipients of beta-blockers was $6.1 \mathrm{pg} / \mathrm{mL}$, while this amount was as high as $3.06 \mathrm{pg} / \mathrm{mL}$ in patients who did not receive any $\beta$-blockers.

Recruiting patients who do not receive $\beta$-blockers is a challenging task. Since in a controlled disease registry system only those with contraindications to $\beta$-blockers were eligible to be enrolled in our study, only 19 patients were fit to be included which accounted as one of the major limitations of this study. Additionally, genetic variants on different isoenzymes of CYP450, mainly CYP2D6 are known to affect serum levels of carvedilol and as a result, may influence clinical outcomes of patients. Existence of various polymorphisms on both target receptors and metabolizing enzymes such as CYP2D6 cause variations in carvedilol serum level as well as its signaling effects, respectively, and hence influence clinical outcomes. ${ }^{34,35}$ Measuring serum levels of carvedilol in both groups of HF patients along with genotyping of CYP2D6 may provide more vivid results in future studies.

Lack of observing association between the cardioprotective effect of carvedilol and serum levels of IL-33 and sST2 between two groups of HF patients is probably due to small sample size of eligible enrolled patients. However, this study may provide a clue for future studies with larger sample sizes.

\section{Conclusion}

Our results showed lack of association between serum levels of IL-33 and sST2 with HF. Additionally, the results did not confirm the cardio protective mechanism of carvedilol by means of IL-33/sST2 pathway.

\section{Ethics Statement}

All participants were informed about the purpose of the study.

\section{Funding}

This work was financially supported by a grant from Pharmaceutical Sciences Research Center, Shiraz University of Medical Sciences, Shiraz, Iran (Grant NO 96-01-36-
15870). The project was a part of a Pharm.D. thesis by Maryam Dashti, Department of Pharmacology and Toxicology, School of Pharmacy, Shiraz University of Medical Sciences, Shiraz, Iran.

\section{Disclosure}

The authors declare that they have no competing interests.

\section{References}

1. Kasper F, Hauser L, Jameson L. Harrison's Principles of Internal Medicine. New York: McGraw-Hill; 2015.

2. Morrissey RP, Czer L, Shah PK. Chronic heart failure. Am J Cardiovasc Drugs. 2011;11(3):153-171. doi:10.2165/11592090000000000-00000

3. Tziakas DN, Chalikias GK, Xatseras DI. Neurohormonal hypothesis in heart failure. Hellenic J Cardiol. 2003;44:195-205.

4. Packer M, Fowler MB, Roecker EB, et al. Effect of carvedilol on the morbidity of patients with severe chronic heart failure: results of the carvedilol prospective randomized cumulative survival (COPERNICUS) study. Circulation. 2002;106(17):2194-2199. doi:10.1161/01.CIR.0000035653.72855.BF

5. Carriere V, Roussel L, Ortega N, et al. IL-33, the IL-1-like cytokine ligand for ST2 receptor, is a chromatin-associated nuclear factor in vivo. Proc Natl Acad Sci U S A. 2007;104(1):282-287. doi:10.1073/ pnas.0606854104

6. Liew FY, Pitman NI, McInnes IB. Disease-associated functions of IL-33: the new kid in the IL-1 family. Nat Rev Immunol. 2010;10 (2):103. doi: $10.1038 /$ nri2692

7. Roussel L, Erard M, Cayrol C, Girard JP. Molecular mimicry between IL-33 and KSHV for attachment to chromatin through the H2A-H2B acidic pocket. EMBO Rep. 2008;9(10):1006-1012. doi:10.1038/embor.2008.145

8. Miller AM, Liew FY. The IL-33/ST2 pathway-a new therapeutic target in cardiovascular disease. Pharmacol Ther. 2011;131 (2):179-186. doi:10.1016/j.pharmthera.2011.02.005

9. Trajkovic V, Sweet MJ, Xu D. T1/ST2 - an IL-1 receptor-like modulator of immune responses. Cytokine Growth Factor Rev. 2004;15 (2-3):87-95. doi:10.1016/j.cytogfr.2004.02.004

10. Schmitz J, Owyang A, Oldham E, et al. IL-33, an interleukin-1-like cytokine that signals via the IL-1 receptor-related protein ST2 and induces T helper type 2-associated cytokines. Immunity. 2005;23 (5):479-490. doi:10.1016/j.immuni.2005.09.015

11. Tago K, Noda T, Hayakawa M, et al. Tissue distribution and subcellular localization of a variant form of the human ST2 gene product, ST2V. Biochem Biophys Res Commun. 2001;285(5):1377-1383. doi:10.1006/bbrc.2001.5306

12. Wood IS, Wang B, Trayhurn P. IL-33, a recently identified interleukin-1 gene family member, is expressed in human adipocytes. Biochem Biophys Res Commun. 2009;384(1):105-109. doi:10.1016/j.bbrc.2009.04.081

13. Pusceddu I, Dieplinger B, Mueller T. ST2 and the ST2/IL-33 signalling pathway-biochemistry and pathophysiology in animal models and humans. Clin Chim Acta. 2019;495:493-500. doi:10.1016/j. cca.2019.05.023

14. Aleksova A, Paldino A, Beltrami AP, et al. Cardiac biomarkers in the emergency department: the role of soluble ST2 (sST2) in acute heart failure and acute coronary syndrome - there is meat on the bone. J Clin Med. 2019;8(2):270. doi:10.3390/jcm8020270

15. Coronado MJ, Bruno KA, Blauwet LA, et al. Elevated sera SST 2 is associated with heart failure in men $\leq 50$ years old with myocarditis. J Am Heart Assoc. 2019;8(2):e008968. doi:10.1161/ JAHA. 118.008968 
16. Lotierzo M, Dupuy AM, Kalmanovich E, Roubille F, Cristol JP. sST2 as a value-added biomarker in heart failure. Clin Chim Acta. 2020;501:120-130. doi:10.1016/j.cca.2019.10.029

17. Sanada S, Hakuno D, Higgins LJ, Schreiter ER, McKenzie AN, Lee RT. IL-33 and ST2 comprise a critical biomechanically induced and cardioprotective signaling system. J Clin Invest. 2007;117 (6):1538-1549. doi:10.1172/JCI30634

18. Human Sst2(Soluble ST2) ELISA Kit; 2017. 7th. Availabe from: www.elabscience.com.

19. Ab119547- IL-33 Human ELISA Kit; 2018. Availabe from: www. abcam.com.

20. Doughty RN, Whalley GA, Gamble G, MacMahon S, Sharpe N. Left ventricular remodeling with carvedilol in patients with congestive heart failure due to ischemic heart disease. J Am Coll Cardiol. 1997;29(5):1060-1066. doi:10.1016/S0735-1097(97)00012-0

21. Group MHS. Effect of metoprolol $\mathrm{CR} / \mathrm{XL}$ in chronic heart failure: metoprolol $\mathrm{CR} / \mathrm{XL}$ randomised intervention trial in-congestive heart failure (MERIT-HF). Lancet. 1999;353(9169):2001-2007. doi:10.1016/S0140-6736(99)04440-2

22. Hall SA, Cigarroa CG, Marcoux L, Risser RC, Grayburn PA, Eichhorn EJ. Time course of improvement in left ventricular function, mass and geometry in patients with congestive heart failure treated with beta-adrenergic blockade. J Am Coll Cardiol. 1995;25 (5):1154-1161. doi:10.1016/0735-1097(94)00543-Y

23. Gaggin HK, Szymonifka J, Bhardwaj A, et al. Head-to-head comparison of serial soluble ST2, growth differentiation factor-15, and highly-sensitive troponin $\mathrm{T}$ measurements in patients with chronic heart failure. JACC Heart Fail. 2014;2(1):65-72. doi:10.1016/j. jchf.2013.10.005

24. Seki K, Sanada S, Kudinova AY, et al. Interleukin-33 prevents apoptosis and improves survival after experimental myocardial infarction through ST2 signaling. Circ Heart Fail. 2009;2(6):684-691. doi:10.1161/CIRCHEARTFAILURE.109.873240

25. Weinberg EO, Shimpo M, De Keulenaer GW, et al. Expression and regulation of ST2, an interleukin-1 receptor family member, in cardiomyocytes and myocardial infarction. Circulation. 2002;106 (23):2961-2966. doi:10.1161/01.CIR.0000038705.69871.D9

26. Shimpo M, Morrow DA, Weinberg EO, et al. Serum levels of the interleukin-1 receptor family member ST2 predict mortality and clinical outcome in acute myocardial infarction. Circulation. 2004;109 (18):2186-2190. doi:10.1161/01.CIR.0000127958.21003.5A
27. Sabatine MS, Morrow DA, Higgins LJ, et al. Complementary roles for biomarkers of biomechanical strain ST2 and N-terminal prohormone B-type natriuretic peptide in patients with ST-elevation myocardial infarction. Circulation. 2008;117(15):1936-1944. doi:10.1161/CIRCULATIONAHA.107.728022

28. Weir RA, Miller AM, Murphy GE, et al. Serum soluble ST2: a potential novel mediator in left ventricular and infarct remodeling after acute myocardial infarction. J Am Coll Cardiol. 2010;55 (3):243-250. doi:10.1016/j.jacc.2009.08.047

29. Bayes-Genis A, Pascual-Figal D, Januzzi JL, et al. Soluble ST2 monitoring provides additional risk stratification for outpatients with decompensated heart failure. Revista española de cardiología. 2010;63(10):1171-1178. doi:10.1016/S1885-5857(10)70231-0

30. Manzano-Fernández S, Mueller T, Pascual-Figal D, Truong QA, Januzzi JL. Usefulness of soluble concentrations of interleukin family member ST2 as predictor of mortality in patients with acutely decompensated heart failure relative to left ventricular ejection fraction. $\mathrm{Am}$ J Cardiol. 2011;107(2):259-267. doi:10.1016/j.amjcard.2010.09.011

31. Miller AM, Xu D, Asquith DL, et al. IL-33 reduces the development of atherosclerosis. J Exp Med. 2012;209(13):2515. doi:10.1084/ jem.2007186820913c

32. Palmer G, Gabay C. Interleukin-33 biology with potential insights into human diseases. Nat Rev Rheumatol. 2011;7(6):321. doi: $10.1038 /$ nrrheum.2011.53

33. Yndestad A, Marshall AK, Hodgkinson JD, Sugden PH, Clerk A. Modulation of interleukin signalling and gene expression in cardiac myocytes by endothelin-1. Int $J$ Biochem Cell Biol. 2010;42 (2):263-272. doi:10.1016/j.biocel.2009.10.021

34. Lymperopoulos A, McCrink K, Brill A. Impact of CYP2D6 genetic variation on the response of the cardiovascular patient to carvedilol and metoprolol. Curr Drug Metab. 2015;17(1):30-36. doi:10.2174/ 1389200217666151105125425

35. Parker BM, Rogers SL, Lymperopoulos A. Clinical pharmacogenomics of carvedilol: the stereo-selective metabolism angle. Future Med. 2018;1089-1093.
Clinical Pharmacology: Advances and Applications is an international, peer-reviewed, open access journal publishing original research, reports, reviews and commentaries on all areas of drug experience in humans. The manuscript management system is completely online and includes a very quick and fair peer-review system, which is all easy to use. Visit http://www.dovepress.com/testimonials.php to read real quotes from published authors. 